\title{
Histiocytosis, a rare cause of hypopituitarism. Langerhans cell histiocytosis and Erdheim-Chester disease, two case reports of pituitary deficiency.
}

Hána V. jr., Kosák M., Hána V.

3rd Department of Internal Medicine, 1st Faculty of Medicine, Charles University and General Teaching Hospital in Prague

Case 1

36-year-old man diagnosed with diabetes insipidus, central hypogonadism, severe $\mathrm{GH}$ deficiency in 2006

on MRI in 2006, 2007, 2009 only absent neurohypophysis signal on $\mathrm{T} 1$, without other pathology

Recurrent Bilateral external otitis since 2011

MRI in 2014: hypothalamic lesion with a pituitary stalk enlargement, multiple white matter lesions in T2W sequences in mesencephalon, pons and cerebellum surrounding the IV. ventricle

2014 dg. Folliculitis capitis by local dermatologist

Histiocyte like cells infiltration of dermis, cells are CD 1a+, S100+, BRAF - in the scalp biopsy

PET/CT showed infiltration of hypothalamus, maxilla, a mandible with perifocal soft tissue involvement and regional lymphadenopathy

Diagnosis: Langerhans cell histiocytosis

Treatment initiated with cladribine (Litak)

\section{Case 2}

42-year-old man with isolated diabetes insipidus, diagnosed in 2014
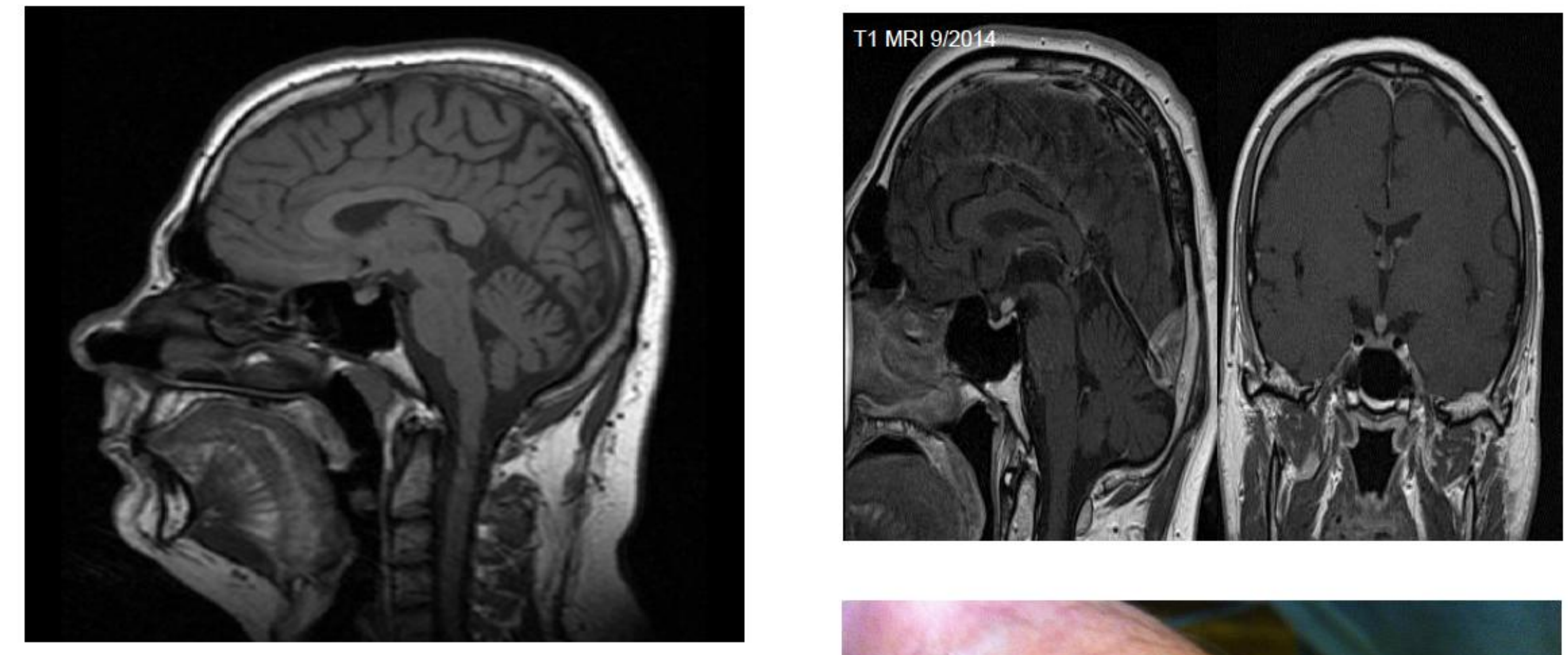

MRI showed an infundibular infiltration of unknown origin
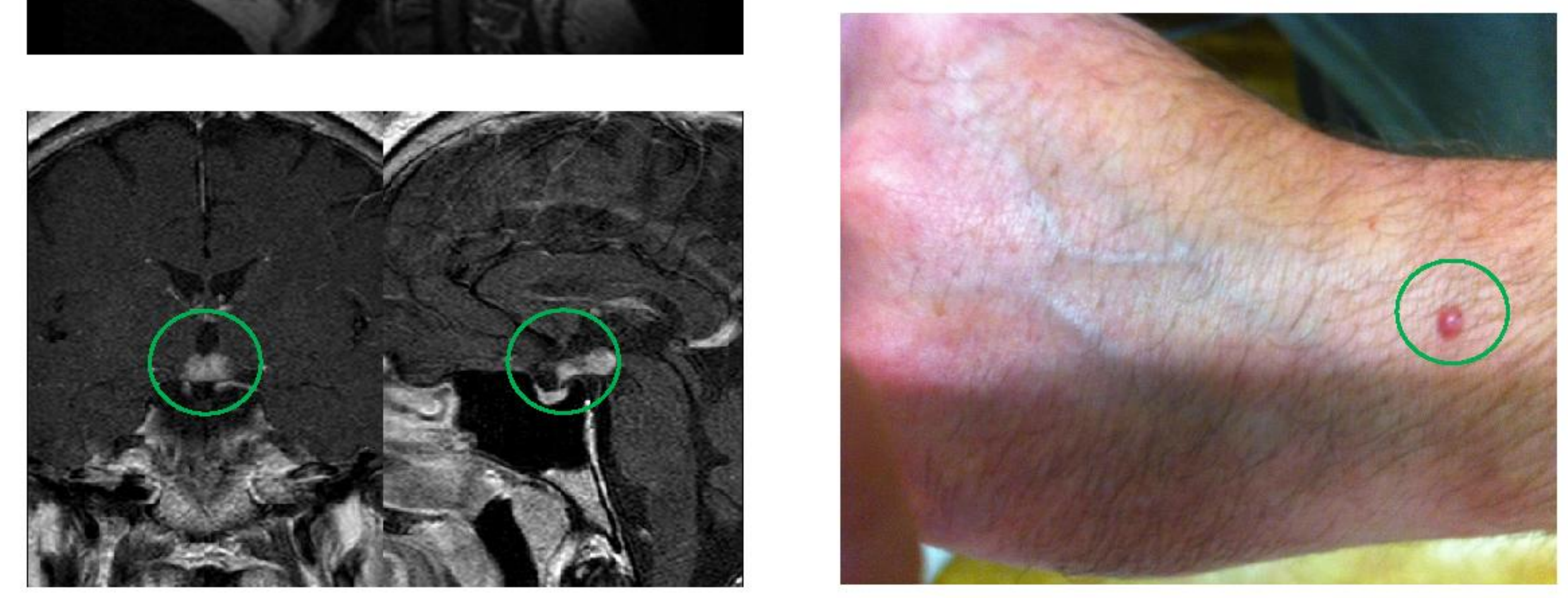

Several skin papules last 5 years, biopsy showed CD 68+, S100-, CD la - histiocytyte infiltrate, not favoring a Langerhans cell histiocytosis, could be a multicentric histiocytosis
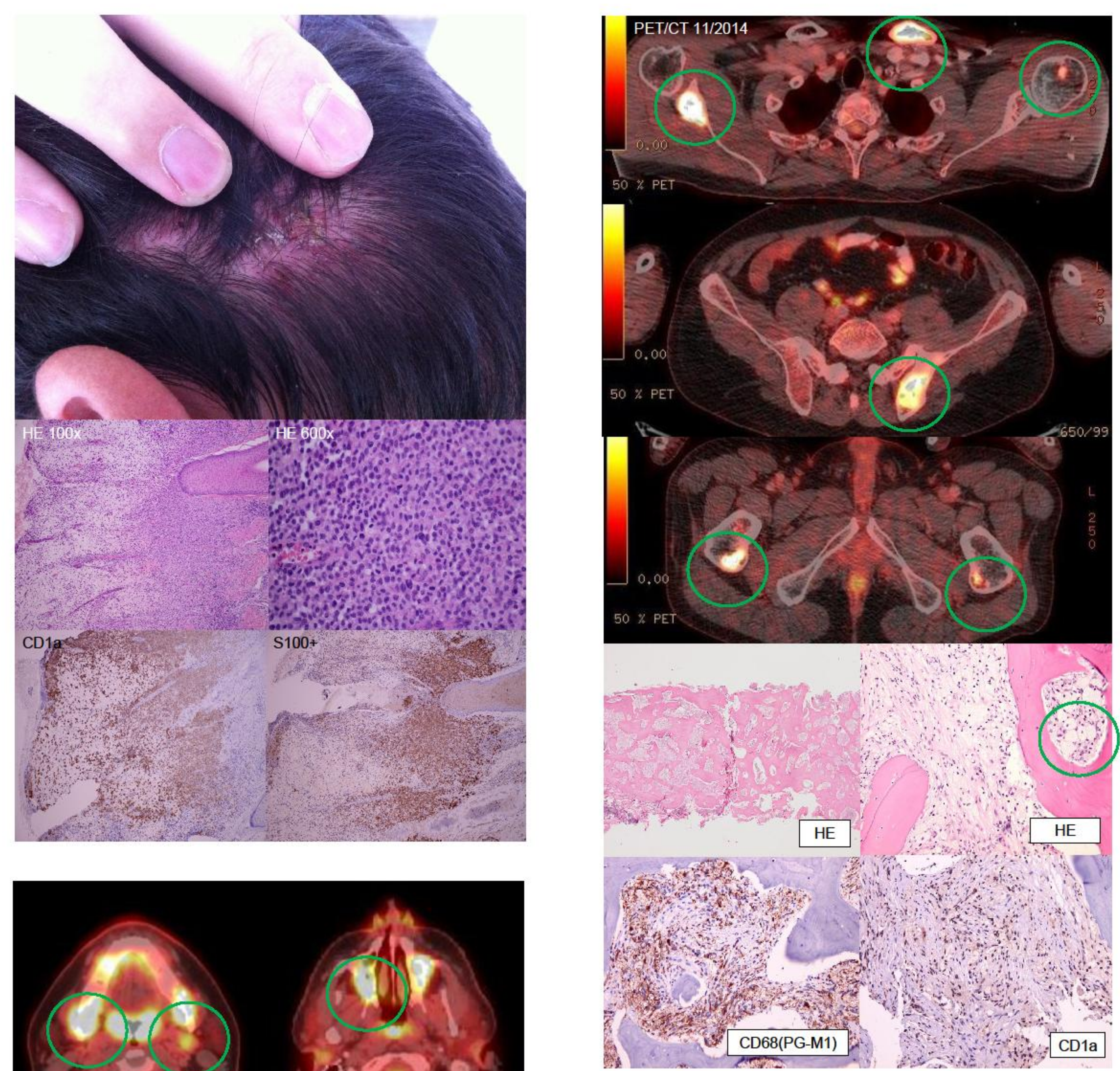

PET/CT showed osteolytic lesions in both scapulas, proximal humeri, clavicles, transversal procesus of Th7, left ilium and proximal parts of femur bones

Clavicle biopsy revealed foamy histiocytes, CD68+, CD 1a and S100-, BRAF +

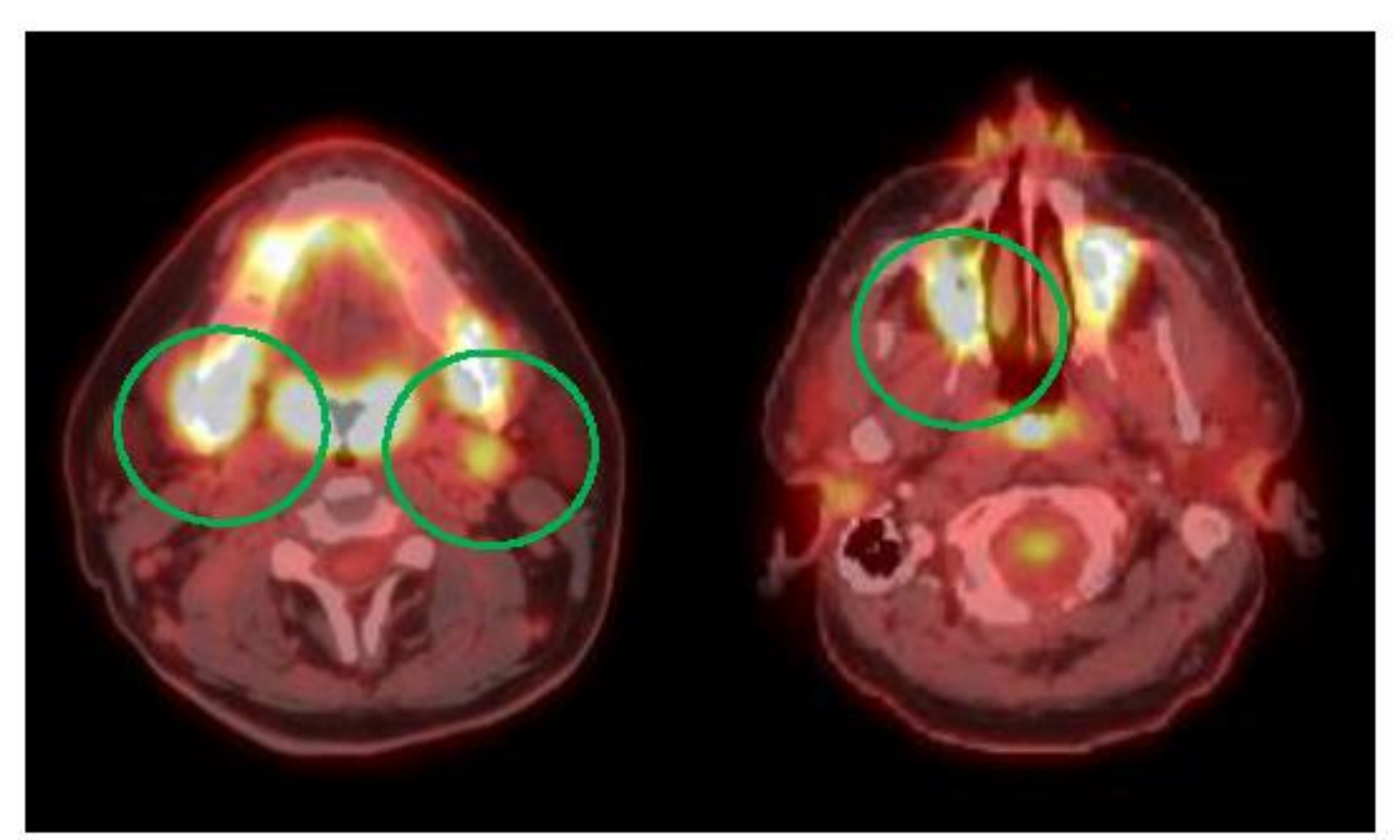

Diagnosis: Erdheim-Chester diesease (nonLangerhans cell histiocytostis)

Treatment iniciated with IFN a (Roferon)

Conclusion: In patients with central diabetes insipidus of unclear etiology precise physical examination and PET/CT scan can reveal a focus appropriate for a biopsy leading to the diagnosis. 